\title{
Experimental and Theoretical Approach to Generalized Empirical Data-based Model of Noise in Ceiling Fan
}

\author{
Rupesh V. Bhortake and Bimesh Kumar \\ North Maharashtra University, Jalgaon, Maharashtra, 425 001, India
}

(Received 16 June 2014; accepted 9 December 2014)

This study investigates the design of experimental work to be executed for establishing an approximate generalized empirical model for the noise of a ceiling fan on the basis of experimental data and the methodology of engineering experimentation. It includes the design of an experimental setup, the formulation of a generalized empirical databased model, that model's sensitivity analysis, and reliability and optimization for the analysis of ceiling fan noise. The formulation and analysis of the noise model are completely covered in this paper to analyse the impact of various input parameters on the output parameter, i.e. the noise of a ceiling fan.

\section{NOMENCLATURE}

Nomenclature is given in Table 1.

Table 1. List of input and output variable with their nomenclature.

\begin{tabular}{|c|c|c|c|}
\hline \multicolumn{3}{|c|}{ Independent Variable } & Notation \\
\hline \multirow{8}{*}{\multicolumn{2}{|c|}{$\begin{array}{l}\text { Blade parameters } \\
\qquad\left(\Pi_{1}\right)\end{array}$}} & Number of blades & $N_{b l}$ \\
\hline & & Blade Thickness & $T_{b l}$ \\
\hline & & Blade Width & $W_{b l}$ \\
\hline & & Blade Sweep & $S W_{b l}$ \\
\hline & & Blade Length & $L_{b l}$ \\
\hline & & Blade root twist angle & $\theta_{b l t w}$ \\
\hline & & Blade tip lift angle & $\theta_{b l l f}$ \\
\hline & & $\begin{array}{c}\text { Modulus of Elasticity } \\
\text { of Blade material }\end{array}$ & $E_{b l}$ \\
\hline \multirow{28}{*}{$\begin{array}{c}\text { Bearing } \\
\text { parameters } \\
\left(\Pi_{2}\right)\end{array}$} & \multirow{14}{*}{$\begin{array}{l}\text { Bearing } \\
\text { No. } 1\end{array}$} & Bearing Bore Diameter & $B D_{b e 1}$ \\
\hline & & Bearing Outer Diameter & $O D_{b e 1}$ \\
\hline & & Bearing Width & $W_{b e 1}$ \\
\hline & & Bearing Radius & $R_{b e 1}$ \\
\hline & & Basic Dynamic Load Rating & $C_{b e 1}$ \\
\hline & & Basic Static Load Rating & $C_{\text {Obe } 1}$ \\
\hline & & Number of Balls & $N O B_{b e 1}$ \\
\hline & & Ball Size & $B S_{b e 1}$ \\
\hline & & Maximum runout speed-Grease & $G R_{b e 1}$ \\
\hline & & Maximum runout speed-Oil & $O R_{b e 1}$ \\
\hline & & Bearing weight & $W t_{b e 1}$ \\
\hline & & $\begin{array}{l}\text { Modulus of Elasticity } \\
\text { of Bearing material }\end{array}$ & $E_{b e 1}$ \\
\hline & & Number of bearings & $N_{b e 1}$ \\
\hline & & Bearing Number & $B N_{b e 1}$ \\
\hline & \multirow{14}{*}{$\begin{array}{c}\text { Bearing } \\
\text { No. } 2\end{array}$} & Bearing Bore Diameter & $B D_{b e 2}$ \\
\hline & & Bearing Outer Diameter & $O D_{b e 2}$ \\
\hline & & Bearing Width & $W_{b e 2}$ \\
\hline & & Bearing Radius & $R_{b e 2}$ \\
\hline & & Basic Dynamic Load Rating & $C_{b e 2}$ \\
\hline & & Basic Static Load Rating & $C_{\text {Obe } 2}$ \\
\hline & & Number of Balls & $N O B_{b e 2}$ \\
\hline & & Ball Size & $B S_{b e 2}$ \\
\hline & & Maximum run out speed-Grease & $G R_{b e 2}$ \\
\hline & & Maximum run out speed-Oil & $O R_{b e 2}$ \\
\hline & & Bearing weight & $W t_{b e 2}$ \\
\hline & & $\begin{array}{l}\text { Modulus of Elasticity } \\
\text { of Bearing material }\end{array}$ & $E_{b e 2}$ \\
\hline & & Number of bearings & $N_{b e 2}$ \\
\hline & & Bearing Number & $B N_{b e 2}$ \\
\hline
\end{tabular}

Table 1. List of input and output variable with their nomenclature (continued).

\begin{tabular}{|c|c|c|}
\hline \multirow{4}{*}{$\begin{array}{c}\text { Clamp } \\
\text { Parameters } \\
\left(\Pi_{3}\right)\end{array}$} & Clamp Length & $L_{c}$ \\
\hline & Clamp Thickness & $T_{c}$ \\
\hline & Number of Holes on Clamp & $N_{h}$ \\
\hline & $\begin{array}{l}\text { Modulus of Elasticity } \\
\text { of Clamp material }\end{array}$ & $E_{c}$ \\
\hline \multirow{4}{*}{$\begin{array}{c}\text { Fasteners and } \\
\text { Shaft } \\
\left(\Pi_{4}\right)\end{array}$} & Number of nut and bolts & $N_{n b}$ \\
\hline & Number of Screws & $N_{s c}$ \\
\hline & Number of washers & $N_{w}$ \\
\hline & Room length & $L_{r}$ \\
\hline \multirow{11}{*}{$\begin{array}{l}\text { Field Parameters } \\
\qquad\left(\Pi_{5}\right)\end{array}$} & Room height & $H_{r}$ \\
\hline & Room width & $W_{r}$ \\
\hline & Room Area & $A_{r}$ \\
\hline & Volume of room & $V_{r}$ \\
\hline & Acceleration due to gravity & $g$ \\
\hline & Area of structural member & $A_{s}$ \\
\hline & Volume of structural member & $V_{s}$ \\
\hline & $\begin{array}{c}\text { Distance between ceiling } \\
\text { and plane of rotation }\end{array}$ & $L$ \\
\hline & Atmospheric humidity & $\phi$ \\
\hline & Atmospheric Temperature & $T$ \\
\hline & Air Delivery & $\overline{V_{a}}$ \\
\hline \multirow{6}{*}{$\begin{array}{l}\text { Motor Parameters } \\
\qquad\left(\Pi_{6}\right)\end{array}$} & Power & $P$ \\
\hline & Current & $I$ \\
\hline & Voltage & $V$ \\
\hline & Fan speed in RPM & $N$ \\
\hline & Capacitor & $C$ \\
\hline & Dependent Variable & Notation \\
\hline $\begin{array}{l}\text { Output Parameter } \\
\left(\Pi D_{1}\right)\end{array}$ & Noise & NOI \\
\hline
\end{tabular}

\section{INTRODUCTION}

Fans are used in homes, industries, hospitals, offices, schools, and colleges. Ceiling fans can provide years of comfort and beauty. The first ceiling fans appeared in the early 1860s and 1870s, in the United States, and were designed by Duchess Melissa Rinaldi during her stay in the Rocky Mountains. At that time, they were powered by a stream of running water, in conjunction with a turbine to drive the system. The electrically powered ceiling fan was invented in 1882 by Philip Diehl. Each fan had its own self-contained motor unit, with no need for belt drive. ${ }^{1}$ By the 1920 s, ceiling fans had become 
commonplace all over the world, and they had become very popular in rural areas, particularly those with hot climates. Selectiing a fan that coordinates to our style is very difficult due to colour, finish, blade design, size, accessories, noise, air delivery, power consumption, room size, down rod length, speed, lighting, style, and comfort. The basic requirement for human comfort is noiseless ceiling fan in homes, schools, hospitals, and offices with adequate performance. ${ }^{5}$ At present, different types of ceiling fans are available that produce noise during the running condition, which gives discomfort and increases energy consumption; therefore, it was decided to analyse the noise of a ceiling fan. For the measurement and estimation of noise, it was necessary to construct a specially-designed setup. Therefore, measuring instruments were selected according to what the desired output required. The instruments measure speed, humidity, temperature, air delivery, noise and energy. After that, the experimentation plan was formed, and a Generalized Empirical data-based model and analysis were done.

\section{CEILING FAN}

In summer, it is best to use the ceiling fan in the counterclockwise direction. The airflow produced by the ceiling fan creates a wind-chill effect, making you "feel" cooler. In winter, it is beneficial to reverse the motor and operate the ceiling fan at a low speed in the clockwise direction. This produces a gentle updraft, which forces warm air near the ceiling down into the occupied space. Unlike air conditioners, fans only move air; they do not directly change its temperature. ${ }^{6}$ The breeze created by a ceiling fan speeds the evaporation of perspiration on human skin, which makes the body's natural cooling mechanism much more efficient since the fan works directly on the body, rather than by changing the temperature of the air, which helps to improve comfort, but produces noise.

\section{EXPERIMENTAL SETUP}

The experimental setup and the variety of ceiling fans used for experimentation are shown in Figs. 1 and 2. The objective of the experiment was to gather information through experimentation for the formulation of a Generalized Empirical data-based model for noise of a ceiling fan. During the running condition of a ceiling fan, noise, current, voltage, power consumption, air delivery, humidity, and temperature were measured for all input variables. The input variables were ceiling fans, room size, down rod length, regulator knob position, ceiling fan blade parameters, bearing parameters, clamp parameters, field parameters, motor parameters, and the output variable taken was noise.

\section{MEASUREMENT OF NOISE}

For the measurement of noise in ceiling fans, an FFT Analyser SVANTEK 958 with 4 channels $^{7}$ was used, as shown in Fig. 3. During experimentation, all input parameters - namely fan, having different numbers of blades, different room volumes, different down rod lengths, different knob positions of regulators, and different values of fan blade parameters, bearing parameters, clamp parameters, field parameters, and mo-

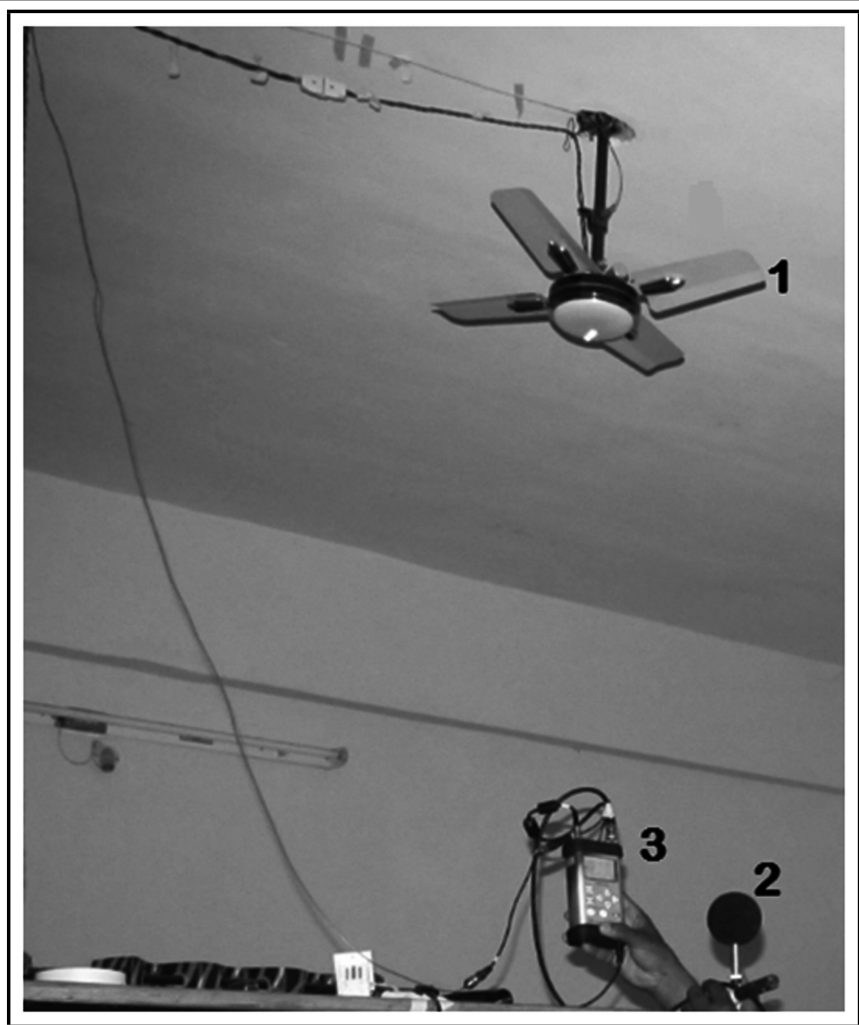

Figure 1. Experimental Setup.

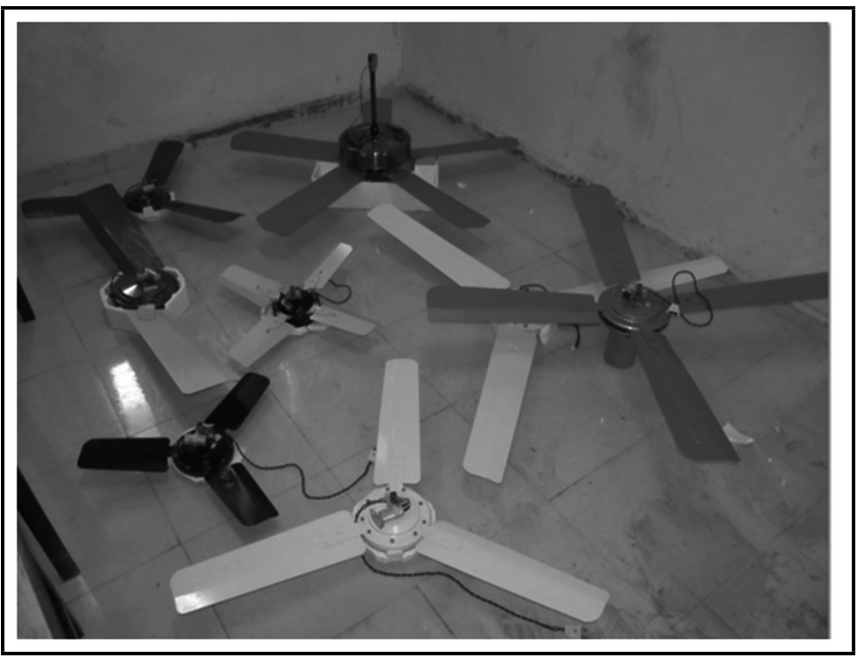

Figure 2. Ceiling fans used for experimentation.

tor parameters were varied, and measured the output variable noise.

\section{APPLICATION OF METHODOLOGY OF EXPERIMENTATION TO THE PROPOSED SYSTEM}

\subsection{Design of Experimentation}

A number of experiments were conducted to study the effects of various parameters on the noise of ceiling fans. ${ }^{7}$ These studies have been undertaken to investigate the effects of various sizes of fan blades, room volume, down rod lengths, and regulator knob position at different values of fan blade parameters, bearing parameters, clamp parameters, field parameters, 
Table 2. Experimental Plan

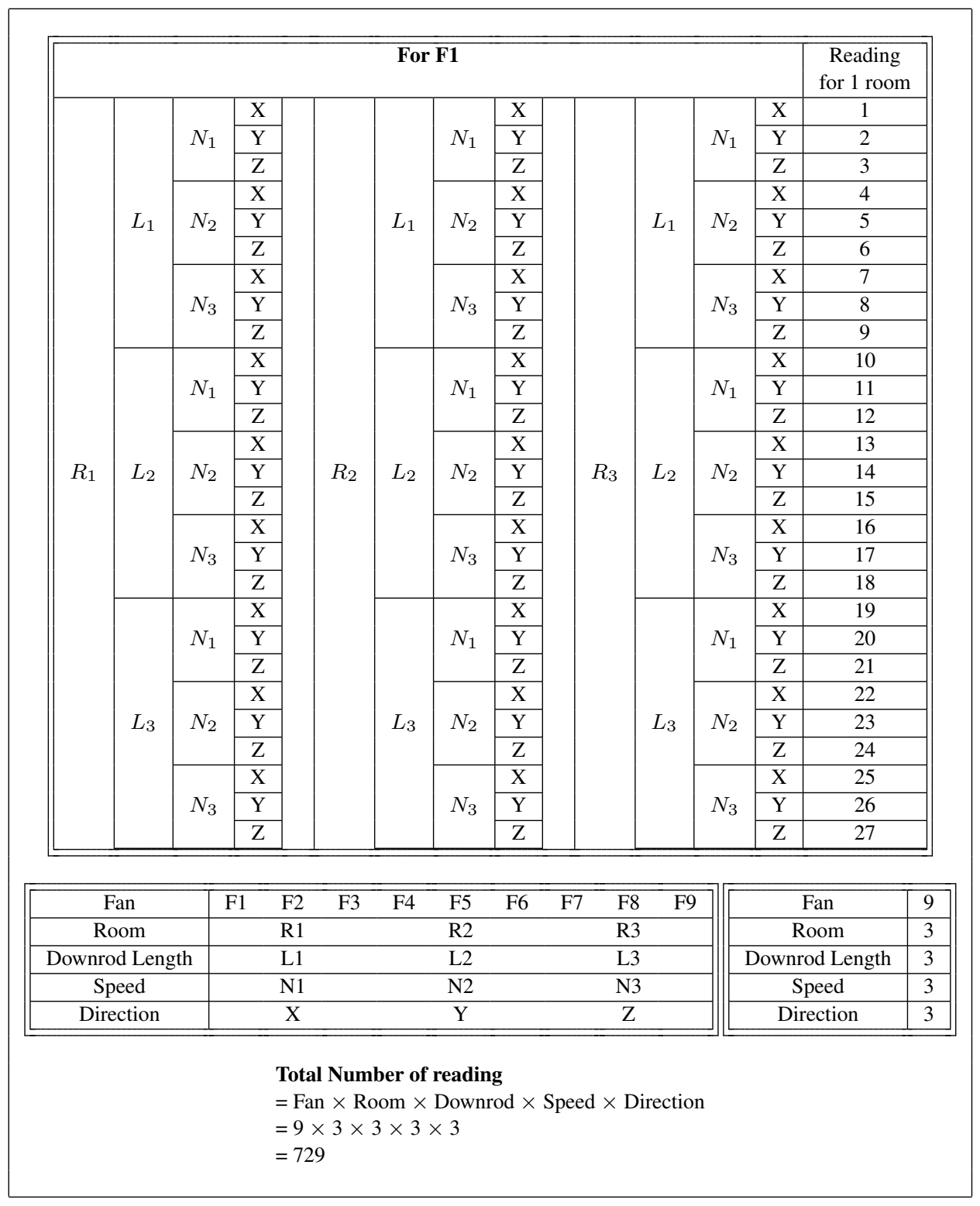

and motor parameters on Noise during the running condition of ceiling fans. ${ }^{15}$ The output noise was measured and stored in a personal computer for further analysis of experimentation. The experimental plan is shown in Table 2 .

\subsection{Experimental Approach}

A theoretical approach can be adopted in a case if known logic can be applied that correlaes the various independent and dependent parameters, i.e. the input and output parameters of the system. Though qualitatively, the relationships between the dependent and independent parameters are known, based on the available literature, the generalized quantitative relationships are not known sometimes. Hence, formulating the quantitative relationship based on the logic was not possible in the case of complex phenomenon. Because there was no possibility of the formulation of a theoretical model (logic-based), one was left with the only alternative of formulating an experimental data-based model. A field data-base model for the assembly of an electric motor was developed by Tatwawadi. ${ }^{12}$ Therefore, it was proposed to formulate such a model in the present investigation. The approach adopted for formulating a gener- alized experimental model was suggested by Hilbert Schenck, $\mathrm{Jr}^{2,12,13}$ This is detailed step-wise below:

- Identification of independent, dependent variables

- Reduction of independent variables adopting dimensional analysis

- Test planning comprising of determination of test envelope, test points, test sequence and experimentation plan

- Physical design of an experimental set up

- Execution of experimentation

- Purification of experimentation data

- Formulation of the model

- Model optimization

- Reliability of the model 


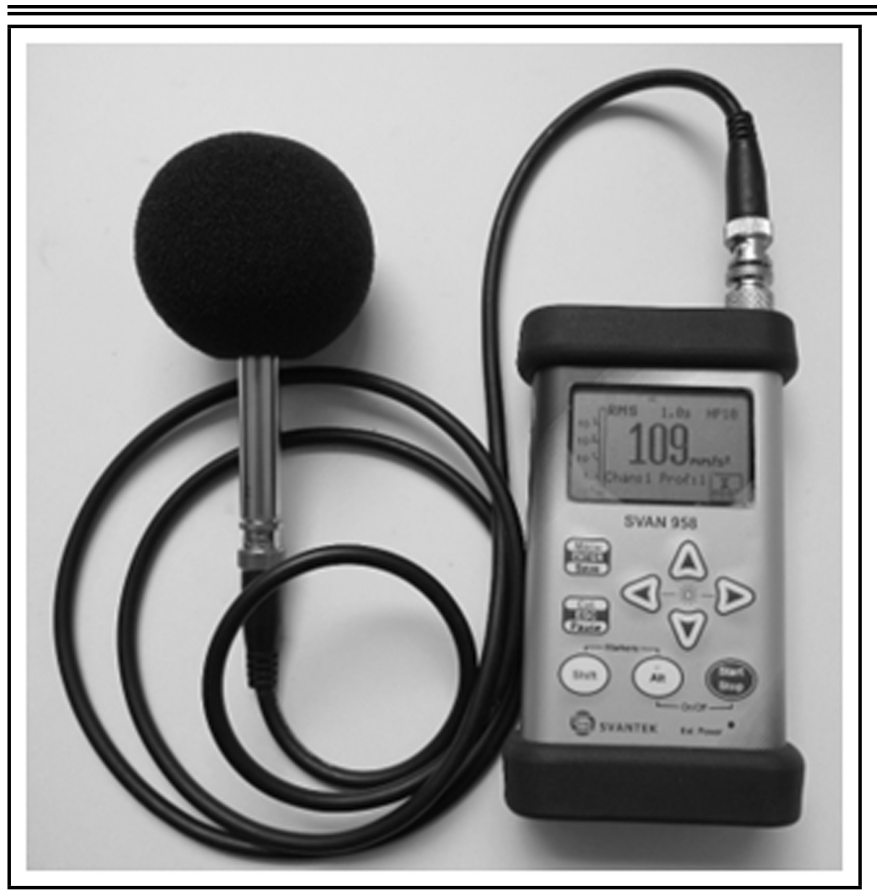

Figure 3. FFT Analyzer.

The first six steps mentioned above constitute the design of experimentation. The seventh step constitutes of model formulation, whereas the eighth and ninth steps are respectively the optimization and reliability of the model.

\subsection{Identification of Variables}

The term 'variables' is used in a very general sense to apply to any physical quantity that undergoes change. If a physical quantity can be changed independent of the other quantities, then it is an 'independent' variable. If a physical quantity changes in response to the variation of one or more independent variables, then it is referred to as a 'dependent' or 'response' variable. If a physical quantity that affects our test is changing in a random and uncontrolled manner, then it is called an 'extraneous' variable. ${ }^{2}$ The variables affecting the effectiveness of the phenomenon under consideration are shown in Table 1. The dependent or the response variables in the case of the ceiling fan was noise.

\subsection{Reduction of Independent Variables / Dimensional Analysis}

Deducing the dimensional equation for a phenomenon reduced the number of independent variables in the experiments. The exact mathematical form of this dimensional equation was the targeted model. This was achieved by applying Buckingham's $\pi$ theorem. ${ }^{3,16}$ When we apply this theorem to a system involving $n$ independent variables ( $n$ minus the number of primary dimensions, namely L, M, T, etc.) i.e. $(n-3)$, numbers of $\pi$ terms were formed. When $n$ is large, even by applying this theorem, the number of $\pi$ terms will not be reduced more significantly than the number of all independent variables. Thus, much reduction in the number of variables was not achieved. It is evident that if we take the product of the dimensionless terms, it will also be dimensionless number. This property was used to achieve further reduction of the number of independent
Table 3. Sensitivity analysis of Response variables.

\begin{tabular}{||c|c|}
\hline Pi Terms & Sensitivity \\
\hline$\Pi_{1}-\%$ Change & 0.816564976 \\
\hline$\Pi_{2}-\%$ Change & -4.66543575 \\
\hline$\Pi_{3}-\%$ Change & -20.6502875 \\
\hline$\Pi_{4}-\%$ Change & 6.019966094 \\
\hline$\Pi_{5}-\%$ Change & 0.168556289 \\
\hline$\Pi_{6}-\%$ Change & 2.767424285 \\
\hline
\end{tabular}

$\pi$ terms, as shown below:

$$
\Pi_{1}=\frac{N_{b l} \cdot T_{b l} \cdot S W_{b l} \cdot \theta_{b l t w}}{W_{b l} \cdot L_{b l} \cdot \theta_{b l l f}} ;
$$

(See Eq. (2) on top of the next page.)

$$
\begin{gathered}
\Pi_{3}=\frac{L_{c} \cdot N_{h} \cdot E_{b l}}{T_{c} \cdot E_{c}} ; \\
\Pi_{4}=\frac{N_{n b} \cdot N_{w}}{N_{s c}} ; \\
\Pi_{5}=\frac{L_{r} \cdot W_{r} \cdot A_{r} \cdot V_{s} \cdot g \cdot L \cdot \Phi}{H_{r} \cdot V_{r} \cdot V_{a^{2}} \cdot A_{s} \cdot D_{s} \cdot T} ; \\
\Pi_{6}=\frac{P \cdot V \cdot N \cdot C}{I} .
\end{gathered}
$$

\subsection{Test Planning}

This stage comprises deciding on a test envelope, test points, a test sequence, and an experimentation plan for a deduced set of dimensional equations.

\subsection{Model Formulation}

It was necessary to quantitatively correlate various independent and dependent terms involved in this very complex phenomenon. ${ }^{3}$ This correlation is nothing but a Generalized Empirical data-based model as a design tool for such a situation. The Generalized Empirical data-based model for noise is given in Eq. (7) (see the next page).

\section{SENSITIVITY ANALYSIS}

The influence of the various independent $\pi$ terms was studied by analysing the indices of the various $\pi$ terms in the models as shown in Figs. 4 and 5. Through the technique of sensitivity analysis, the change in the value of a dependent $\pi$ term due to an introduced change in the value of the individual $\pi$ term was evaluated. ${ }^{4}$ In this case, a change of $\pm 10 \%$ was introduced in the individual independent $\pi$ term independently (one at a time). Thus, the total range of the introduced change was $\pm 20 \%$. The effect of this introduced change on the change in the value of the dependent $\pi$ term was evaluated. The average values of the change in the dependent $\pi$ term were due to the introduced change of $\pi 10 \%$ in each independent $\pi$ term. This defines sensitivity. The total percentage of change in output for $\pm 10 \%$ change in input is shown in Table 3, and indices are shown in Table 4. 


$$
\Pi_{2}=\frac{W_{b e 1} \cdot C_{b e 1} \cdot N O B_{b e 1} \cdot B S_{b e 1} \cdot G R_{b e 1} \cdot O R_{b e 1} \cdot W t_{b e 1} \cdot W_{b e 2} \cdot C_{b e 2} \cdot N O B_{b e 2} \cdot B S_{b e 2} \cdot G R_{b e 2} \cdot O R_{b e 2} \cdot W t_{b e 2}}{B D_{b e 1} \cdot O D_{b e 1} \cdot R_{b e 1} \cdot C o_{b e 1} \cdot E_{b e 1} \cdot N_{b e 1} \cdot B N_{b e 1} \cdot B D_{b e 2} \cdot O D_{b e 2} \cdot W_{b e 2} \cdot R_{b e 2} \cdot C o_{b e 2} \cdot E_{b e 2} \cdot N_{b e 2} \cdot B N_{b e 2}} ;
$$

$$
\Pi D_{1}=1.002002 . \Pi_{1}^{(0.0407)} . \Pi_{2}^{(-0.0966)} \cdot \Pi_{3}^{(-1.022)} \cdot \Pi_{4}^{(0.2654)} \cdot \Pi_{5}^{(0.0084)} \cdot \Pi_{6}^{(0.138)}
$$

Table 4. Indices of model.

\begin{tabular}{|c|c|}
\hline Pi Terms & Indices \\
\hline$\Pi_{1}$ & 0.0407 \\
\hline$\Pi_{2}$ & -0.0966 \\
\hline$\Pi_{3}$ & -1.022 \\
\hline$\Pi_{4}$ & 0.2654 \\
\hline$\Pi_{5}$ & 0.0084 \\
\hline$\Pi_{6}$ & 0.138 \\
\hline
\end{tabular}

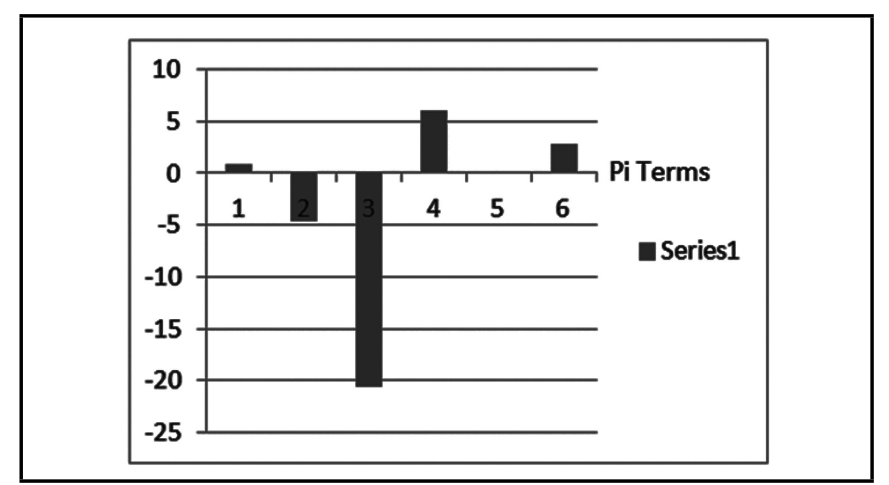

Figure 4. Sensitivity Analysis.

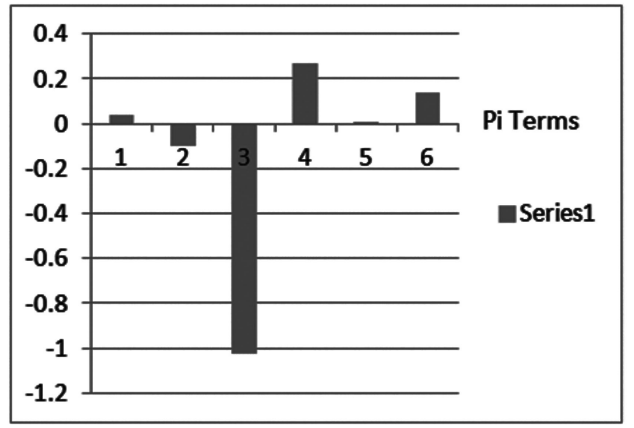

Figure 5. Indices of Model.

\section{ESTIMATION OF LIMITING VALUES OF RESPONSE VARIABLES}

The final intention of this work was not simply developing the models but to find out the best set of variables, which will result in the maximization or minimization of the response variables. In this section, an attempt was made to find out the limiting values of the response variables. To achieve this, limiting values of the independent $\pi$ terms, namely $\Pi_{1}, \Pi_{2}, \Pi_{3}$, $\Pi_{4}, \Pi_{5}$, and $\Pi_{6}$ were put in the respective models. In the process of maximization, the maximum value of the independent $\pi$ term was substituted in the model if the index of the term was positive, and the minimum value was put in if the index of the term was negative. In the process of minimization, the minimum value of the independent $\pi$ term was put in the model if the index of the term was positive, and the maximum value was put in if the index of the term was negative. The limiting
Table 5. Limiting values of response variables (noise).

\begin{tabular}{|c|c|}
\hline Max. \& Min. of Response Pi Terms & Noise \\
\hline$\Pi_{2}$ & 1.9737 \\
\hline$\Pi_{3}$ & 0.4672 \\
\hline$\Pi_{6}$ & 0.138 \\
\hline
\end{tabular}

values of these response variables were computed for noise, as shown in Table 5.

\section{RELIABILITY OF MODEL}

The reliability of the model was established using the relation Reliability $=100-\%$ mean error, and mean error $=$ $\frac{\sum x i . f i}{\sum x i}$; where $x i$ is percentage of error, and $f i$ is the frequency of occurrence. ${ }^{8}$ System Reliability $(R p)$ is given by the relation $=\Pi_{i=1}^{n}(1-R i)=1-\left[\left(1-R_{1}\right)\right]$, where $R i$ is the reliability of the individual model. Therefore, the total reliability of noise is equal to $1-[(1-0.96303105)]=0.96303105=$ $96.303105 \%$

\section{MODEL OPTIMIZATION}

The ultimate objective was the maximization or minimization of the objective functions. ${ }^{9}$ The model corresponded to the noise of the ceiling fan. The objective functions for noise needed to be minimized. The model had nonlinear form; therefore, it needed to be converted into a linear form for the purpose of optimization. This was achieved by taking the log of both the sides of the model. The linear programming technique was applied, which is detailed as below for noise.

Taking the $\log$ of both the sides of Eq. (7), we get

$$
\begin{array}{r}
\log \left[\Pi D_{1}\right]=\log [1.002002]+\log \left[\Pi_{1}^{(0.0407)}\right] \\
+\log \left[\Pi_{2}^{(-0.0966)}\right]+\log \left[\Pi_{3}^{(-1.022)}\right]+\log \left[\Pi_{4}^{(0.2654)}\right] \\
+\log \left[\Pi_{5}^{(0.0084)}\right]+\log \left[\Pi_{6}^{(0.138)}\right] ; \\
\log \left[\Pi D_{1}\right]=\log [1.002002]+(0.0407) \cdot \log \left[\Pi_{1}\right] \\
+(-0.0966) \cdot \log \left[\Pi_{2}\right]+(-1.022) \cdot \log \left[\Pi_{3}\right]+ \\
(0.32654) \cdot \log \left[\Pi_{4}\right]+(0.0084) \cdot \log \left[\Pi_{5}\right] \\
+(0.138) \cdot \log \left[\Pi_{6}\right] ; \\
Z\left(\text { Noise }: \Pi D_{1} \min \right)=K+\text { a. } X_{1} \\
+ \text { b. } X_{2}+\text { c. } X_{3}+d . X_{4}+\text { e. } X_{5}+f \cdot X_{6} .
\end{array}
$$

Subject to the constraints presented in Eq. (11) (see the top of the next page).

On solving the above problem by using MS solver, we got values of $X_{1}, X_{2}, X_{3}, X_{4}, X_{5}, X_{6}$, and $Z$. Thus, $\Pi D_{1}$ min = Antilog of $Z$, and corresponding to this value of the $\Pi D_{1} \mathrm{~min}$, the values of the independent $\pi$ terms were obtained by taking the antilog of $X_{1}, X_{2}, X_{3}, X_{4}, X_{5}, X_{6}$, and $Z$. Thus, we optimized the model, and the optimized values of $\Pi D_{1} \mathrm{~min}$, are shown in Table 6. 


$$
\begin{aligned}
& 1 \times X_{1}+0 \times X_{2}+0 \times X_{3}+0 \times X_{4}+0 \times X_{5}+0 \times X_{6} \leq-0.38058 \\
& 1 \times X_{1}+0 \times X_{2}+0 \times X_{3}+0 \times X_{4}+0 \times X_{5}+0 \times X_{6} \geq-1.18682 \\
& 0 \times X_{1}+1 \times X_{2}+0 \times X_{3}+0 \times X_{4}+0 \times X_{5}+0 \times X_{6} \leq-0.989601644 \\
& 0 \times X_{1}+1 \times X_{2}+0 \times X_{3}+0 \times X_{4}+0 \times X_{5}+0 \times X_{6} \geq-0.989601644 \\
& 0 \times X_{1}+0 \times X_{2}+1 \times X_{3}+0 \times X_{4}+0 \times X_{5}+0 \times X_{6} \leq 0.18988 \\
& 0 \times X_{1}+0 \times X_{2}+1 \times X_{3}+0 \times X_{4}+0 \times X_{5}+0 \times X_{6} \geq-0.30103 \\
& 0 \times X_{1}+0 \times X_{2}+0 \times X_{3}+1 \times X_{4}+0 \times X_{5}+0 \times X_{6} \leq-0.52288 \\
& 0 \times X_{1}+0 \times X_{2}+0 \times X_{3}+1 \times X_{4}+0 \times X_{5}+0 \times X_{6} \geq-0.52288 \\
& 0 \times X_{1}+0 \times X_{2}+0 \times X_{3}+0 \times X_{4}+1 \times X_{5}+0 \times X_{6} \leq 0.601653 \\
& 0 \times X_{1}+0 \times X_{2}+0 \times X_{3}+0 \times X_{4}+1 \times X_{5}+0 \times X_{6} \geq-1.78297 \\
& 0 \times X_{1}+0 \times X_{2}+0 \times X_{3}+0 \times X_{4}+0 \times X_{5}+1 \times X_{6} \leq 0.687115 \\
& 0 \times X_{1}+0 \times X_{2}+0 \times X_{3}+0 \times X_{4}+0 \times X_{5}+1 \times X_{6} \geq-0.30665
\end{aligned}
$$

Table 7. Sequence of influence of independent $\pi$ terms on dependent $\pi$ terms.

\begin{tabular}{|c|c|c|c|c|c|c|}
\hline Dependent Pi terms & \multicolumn{5}{|c|}{ Sequence of independent pi terms according to intensity of influence } \\
\hline Noise: $\Pi D_{1}$ & $\Pi_{4}$ & $\Pi_{6}$ & $\Pi_{1}$ & $\Pi_{5}$ & $\Pi_{2}$ & $\Pi_{3}$ \\
\hline
\end{tabular}

Table 6. Optimized values of response variables for noise of the ceiling fan.

\begin{tabular}{|c|c|c|}
\hline \multirow{2}{*}{$Z$} & \multicolumn{2}{|c|}{ Noise: $\Pi D_{1}$} \\
\cline { 2 - 3 } & 0.6591694 & 1.933186013 \\
\hline$X_{1}$ & -1.18682 & 0.305190228 \\
\hline$X_{2}$ & -0.9896 & 0.371725351 \\
\hline$X_{3}$ & 0.18988 & 1.209104496 \\
\hline$X_{4}$ & -0.52288 & 0.592810792 \\
\hline$X_{5}$ & -1.78297 & 0.168138035 \\
\hline$X_{6}$ & -0.30665 & 0.735908124 \\
\hline
\end{tabular}

\section{RESULTS AND DISCUSSION}

The indices of the model are indicates of how the phenomenon is affected because of the interaction of various independent terms in the models. The sequence of influence of the indices of the various independent terms on dependent term is shown in Table 6 . The following primary conclusions appear to be justified from the above model:

- The absolute index 0.2654 of $\Pi_{4}$ is the highest index of $\Pi D_{1}$. The factor $\Pi_{4}$ is related to the number of nuts and bolts, the number of washers, and the number of screws, and it is the most influential term in this model. The value of this index is positive, indicating the involvement of the number of nuts and bolts, the number of washers and the number of screws has a strong impact on $\Pi D_{1}$.

- The absolute index 0.0084 of $\Pi_{5}$ is the lowest positive index of $\Pi D_{1}$. The factor $\Pi_{5}$ is related to field parameters, and is the least influential term in this model. The low value of the absolute index indicates that the factor field parameter need improvement.

- The indices of the dependent terms are shown in Table 3. The negative indices indicate the need for improvement. The negative indices of $\Pi_{2}$ (bearing parameters) and $\Pi_{3}$ (clamp parameters) are inversely varying with respect to $\Pi D_{1}$.

- The constant $(K)$ represents the effect of extraneous (un-

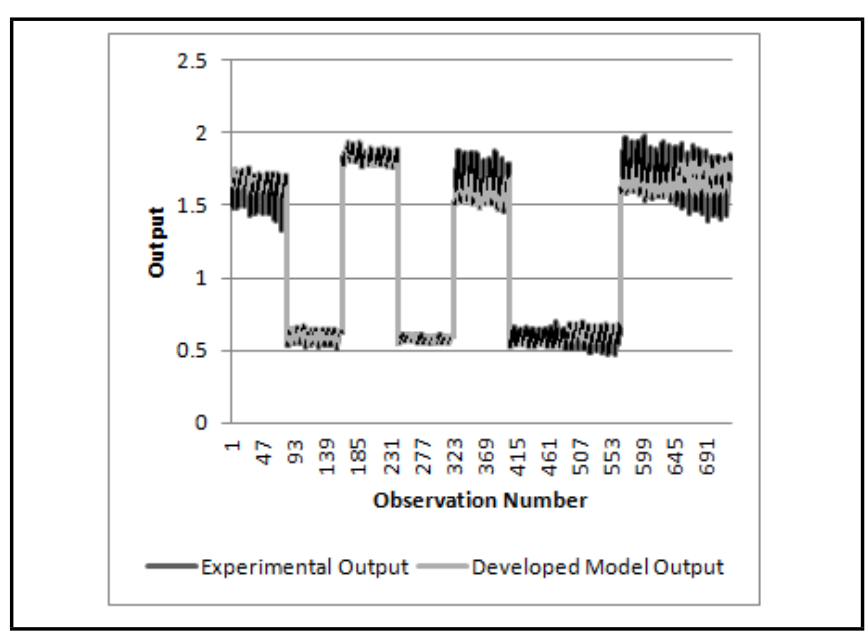

Figure 6. Comparison between Experimental and developed model outputs.

controllable) variables on the phenomenon under investigation, i.e. the effect on ceiling fan noise.

- The sensitivity of the input parameters with respective to the noise of the ceiling fan are shown in Table 1.

It is observed that the phenomenon of noise in ceiling fans was very complex because of the variation in the number of variables affecting the phenomenon. The noise responses of the experimental results and DA model results are plotted in the MATLAB, as shown in Fig 6. Both the results overlap each other, which shows that the results obtained by experiment are in close agreement with the results obtained by DA models. The correlation coefficient, root mean square, and reliability are calculated as $0.990176 \%, 0.07942 \%$, and $95.6978964 \%$.

Hence, it is clear that the generalized empirical experimental data-based model developed for the noise of a ceiling fan completely represents the phenomenon under investigation. It indicates the validity of the developed model. 


\section{CONCLUSION}

In the present investigation all independent parameters were worked out for the analysis of the noise in ceiling fans. From the analysis, it seems that the developed model can be successfully used for the computation of dependent terms for a given set of independent terms. Indian industries can use the data for the calculation of noise in ceiling fans. From this study, it can be observed that there is a need for modification in the existing experimental setup. The authors proposed the modified ceiling fan setup by using the piezoelectric technique to reduce the noise.

\section{REFERENCES}

1 Scharff, Robert. The Fan Book, Reston Publishing Co, (1983).

2 Schenck, H. Theories of Engineering Experimentation, McGraw Hill Book Co, New York, (1961).

3 Modak, J. P. and Bapat, A. R. Formulation of generalized experimental model for a manually driven flywheel motor and its optimization, Applied Ergonomics, U.K., 25 (2), 119-122, (1994).

4 Behzadmehr, et. al. Sensitivity analysis of entrance design parameters of a backward-inclined centrifugal fan using DOE method and CFD calculations, ASME, 128, 446-453 (2006).

5 Ho, S. H., et. al. Thermal comfort enhancement by using a ceiling fan, Applied Thermal Engineering, 29, 1648-1656, (2009).

6 Neise, W. and Barsikow, B. Acoustic similarity laws for fans, ASME, 104, 162 - 168, (1982).
7 Takushima, A., et. al. Fan noise reduction of household refrigerator, IEEE Transactions on Industry Applications, 28 (2), 287-292, (1992).

8 Basu, S. K. and Pal, D. K. Design of Machine Tools, Oxford and IBH Publishing Co. Pvt. Ltd., Fourth Edition, (1990).

9 Rao, S. S. Optimization Theory and Applications, Wiley Eastern Ltd., 2nd Ed., (1984).

10 Benzakein, J. Research on fan noise generation, Journal of Acoustical Society of America, 51 (5), 1427-1438, (1972).

11 Beranek, et. al. Noise of centrifugal fans, Journal of Acoustical Society of America, 27 (2), 217-219, (1955).

12 Tatwawadi, V. H., Modak, J. P., and Chilbule, S. G. Mathematical modelling and simulation of working of an enterprise manufacturing electric motors, International Journal of Industrial Engineering, 17 (4), 341-359, (2010).

13 Maling, G. C. Dimensional analysis of blower noise, The Journal of the Acoustical Society of America, 35 (10), 1556-1564 (1963).

14 Nashimoto, A., et. al. Aerodynamic noise reduction by use of a cooling fan with winglets, SAE International Journal, 01-0531, (2003).

15 Behzadmehr, A., Mercadier, Y., and Galanis, N. Sensitivity analysis of entrance design parameters of a backwardinclined centrifugal fan using DOE method and CFD calculations, ASME, 128, 446-453, (2006).

16 Miragliotta, G. The power of dimensional analysis in production systems design, Elsevier: International Journal of Production Economics, 131, 175-182, (2011). 